\title{
Cadmium phosphide as a new material for infrared converters
}

\author{
D. Stepanchikov ${ }^{1}$, S. Shutov ${ }^{2}$ \\ ${ }^{1}$ Kherson National Technical University, Department of General and Applied Physics \\ 24, Berislavskoye shosse, 73008 Kherson, Ukraine \\ E-mail: step_75@mail.ru; phone:+380(552) 326922 \\ ${ }^{2} V$. Lashkaryov Institute of Semiconductor Physics, NAS of Ukraine \\ 41, prospect Nauky, 03028 Kyiv, Ukraine \\ E-mail: shutov_sv@mail.ru,phone/fax: +380(552) 515457
}

\begin{abstract}
Possible use of cadmium phosphide $\left(\mathrm{Cd}_{3} \mathrm{P}_{2}\right)$ for infrared converter systems has been debated. The interband absorption coefficient calculations has been executed for single crystals of $n-\mathrm{Cd}_{3} \mathrm{P}_{2}$ and interpreted in the exact generalized Kildal band model. Relationship between the absorption coefficient and radiation temperature is presented. On the ground of our calculations, the theoretical temperature dependences of the maximum values of photovoltage and efficiency have been obtained. A common thermodynamical approach was applied in this case. The source of radiation was a black body. In our investigations, the barrier structure on metal-semiconductor basis with the Schottky layer has been considered. The operation temperature range for the $\mathrm{Me}-n-\mathrm{Cd}_{3} \mathrm{P}_{2}$ converter has been found.
\end{abstract}

Keywords: cadmium phosphide, interband absorption, infrared converter.

Manuscript received 29.06.06; accepted for publication 23.10.06.

\section{Introduction}

In recent years, perceptible interest has been focused on the two phosphorus compounds belonging to the crystalline class $\mathrm{A}_{3}^{\mathrm{II}} \mathrm{B}_{2}^{\mathrm{V}}: \mathrm{Zn}_{3} \mathrm{P}_{2}$ and $\mathrm{Cd}_{3} \mathrm{P}_{2}$. It is caused by their excellent prospects for optoelectronic applications. For instance, cadmium phosphide is well known as the infrared laser active medium. Besides, it may be considered as an infrared converter or sensor due to the suitable bandgap: $E_{g}=0.53 \mathrm{eV}$.

The exceptionally complicated crystal structures and the large unit cells are characteristic for the $\mathrm{A}_{3}^{\mathrm{II}} \mathrm{B}_{2}^{\mathrm{V}}$ compounds. For example, both crystals may have a primitive tetragonal lattice with the unit cell consisting of $16 \mathrm{P}$ atoms and 24 metallic atoms. Their space group is $D_{4 h}^{15}$. Thereto, presence of the structural vacancies is natural for these crystalline lattices. Each metallic atom (cadmium, for instance) is in tetrahedral coordination with four phosphorus atoms as with own nearest neighbors, while a phosphorus atom is surrounded by metallic atoms located only at six of the eight corners of the coordination cube so that two vacant sites are placed at the diagonally opposed corners of this cube [1].
Cadmium phosphide always possessed and possesses hitherto only the $n$-type conductivity. Typical concentrations of carriers for as-grown samples are $10^{17} \ldots 10^{18} \mathrm{~cm}^{-3}$. The Fermi level is located somewhat above the bottom of the conduction band. As a result, cadmium phosphide is a degenerate $n$-type semiconductor.

In this paper, the authors examined such converter parameters as the maximum values of the photovoltage and efficiency in dependence on the radiation temperature. These evaluations need the accurate calculations of the absorption coefficient. This coefficient ought to be computing here for $\mathrm{Cd}_{3} \mathrm{P}_{2}$ within the recent generalization of the Kildal band model [2-4].

\section{Band structure}

The dispersion law (Hamiltonian) for $\mathrm{A}_{3}^{\mathrm{II}} \mathrm{B}_{2}^{\mathrm{V}}$ compounds has the following form within the model [2], if to apply the spherical system of coordinates $(k, \theta, \varphi)$ :

$$
\begin{aligned}
& \left(\Gamma(\varepsilon)-(P k)^{2}\left(f_{1}(\varepsilon) \sin ^{2} \theta+f_{2}(\varepsilon) \cos ^{2} \theta\right)\right)^{2}- \\
& -(P k)^{2} f_{3}^{2}(\varepsilon) \sin ^{2} \theta=0 .
\end{aligned}
$$


There $\Gamma(\varepsilon), f_{1}(\varepsilon), f_{2}(\varepsilon), f_{3}(\varepsilon)$ are the known polynomials for the carrier energy and some material parameters. There are no polynomials among them, which exceed the fourth power.

The material parameters of this model are the following five. At first, $E_{g}, \Delta, P$ are three well-known Kane's parameters [3] (i.e., the energy gap, the spinsplitting parameter and the matrix element of the momentum). Further, $\delta$ is the parameter of the tetragonal crystal field [4], and $d$ is the extra field parameter describing the absence of the symmetry center [2]. This additional is equal to zero, if the crystal has a symmetry center. Finally, $\eta$ is the scalar factor taking into account the deformation of the lattice [2]. The energy is measured from the heavy holes band top. Since Eq. (1) does not contain the spherical angle $\varphi$, the surfaces of equal energies are rotation surfaces around the crystal main axis that is also the polar axis.

Obviously that Eq. (1) has the simple decomposition into product of two factors. Both factors should be even identical, if the crystal has a centre of symmetry (herewith $d=0$ and $\left.f_{3}(\varepsilon)=0\right)$. Since it is valid for above mentioned space group $D_{4 h}^{15}$, thus these four solutions of Eq. (1) indeed were found (as functions $\varepsilon(k, \theta))$ under this condition. In Fig. 1, the curves in accord with these solutions are plotted for the conduction band (c), heavy holes (v1), light holes (v2) and spin-orbit splitting (v3) bands.

\section{Interband absorption coefficient}

Fig. 1 illustrates the band structure of $\mathrm{Cd}_{3} \mathrm{P}_{2}$ with the set of the material parameters given in Section 2. All band extremes are located at $\Gamma$ point $(k=0)$. The direct interband transitions are most probable in this case for the photon energy $h v \geq E_{g}$. Fig. 1 shows six possible direct interband transitions labelled A, B, C, D, G and $\mathrm{H}$. The realization of transitions $\mathrm{A}, \mathrm{B}$ and $\mathrm{C}$ causes the generation of free carriers, which can participate in initiating the converter photocurrent.

The work [5] gives the absorption coefficient $K_{i j}$ for each transition from a band $i$ to other band $j$ as follows:

$$
K_{i j}=\frac{\pi e^{2}}{\varepsilon_{0} n c m_{0}^{2} \omega} M_{i j}^{2} \rho_{i j} f_{i j} .
$$

Here $\omega$ is the photon frequency, $\varepsilon_{0}$ is the electric constant, $m_{0}$ is the free electron mass, $n$ is the refraction index of $\mathrm{Cd}_{3} \mathrm{P}_{2}$ and $c$ is the velocity of the light. $M_{i j}$ and $\rho_{i j}$ are the momentum matrix element and the joint density of states between bands $i$ and $j$, respectively. Because $\mathrm{Cd}_{3} \mathrm{P}_{2}$ is an $n$-type degenerated material, the factor $f_{i j}$ takes into account the distribution of unoccupied states in the band $j$ and the occupied ones in the band $i$. The full photon energies $h v$ in the absorption processes of type A, B and C satisfy the condition: $h v>>k_{0} T$. Therefore, the realistic approach may consider the lowest $i$-band as wholly filled. Accordingly, just the statistics of unoccupied states of the highest $j$-band remains to be taken into account. Now, the factor $f_{i j}$ coincides with the Fermi-Dirac distribution [6]:

$$
f_{i j}=1-\left\{1+\exp \left[\frac{\varepsilon_{j}-E_{\mathrm{F}}}{k_{0} T}\right]\right\}^{-1} .
$$

Here $T$ is the temperature of materials, $k_{0}$ is Boltzmann's constant, $\varepsilon_{j}$ is an energy level in the $j$-band. Actually, the transitions between the valence bands D, G, H (Fig. 1) will take place only after beginning of the transitions $\mathrm{A}$ and $\mathrm{B}$. The current calculations relate to $E_{g} \leq E \leq 1.7 E_{g}$ photon energy interval.

The momentum matrix element and the joint density of states have been calculated by the method [5]. To be scrupulous, the total interband absorption coefficient $K$ must be determined by summing up all the possible contributions:

$K=K_{A}+K_{B}+K_{C}+K_{D}+K_{G}+K_{H}$.

Conversely, the part of contributions (primarily D, $\mathrm{G}, \mathrm{H})$ may be omitted keeping a well approximation. Although because the main contributions in absorption are made by interband transitions $(\mathrm{A}, \mathrm{B}, \mathrm{C})$, i.e., between all the valence bands and conduction one. It looks realistic for the investigated photon energy band at least.

\section{Photovoltage and efficiency}

Since the $p$-type samples of $\mathrm{Cd}_{3} \mathrm{P}_{2}$ are yet unattainable, here just the barrier structure between metal and semiconductor is considered. The following admissions are accepted herewith: 1) the diode theory is equitable; 2) the space-charge region looks like the Schottky layer; 3) the tunnel effect is negligible.

The photovoltage may be calculated through the account of the change in the surface potential on irradiation [7]:

$U_{s}=\phi_{s}^{*}-\phi_{s}$.

Here $\phi_{S}$ is the surface potential in the absence of irradiation. The magnitude $\phi_{s}=2 / 3 E_{g}$ is in use further. It corresponds to the maximum value of barrier for the most of such systems on a uniform substrate [8].

The surface potential on irradiation $\phi_{s}^{*}$ is available from the Johnson equation [7]: 
$\delta_{p}=\frac{\exp \left(-Y_{S}\right)-\exp \left(-Y_{S}^{*}\right)+\lambda^{-2}\left(\exp \left(Y_{S}\right)-\exp \left(Y_{S}^{*}\right)\right)+\left(1-\lambda^{-2}\right)\left(Y_{S}-Y_{S}^{*}\right)}{\exp \left(-Y_{S}^{*}\right)+\exp \left(Y_{S}^{*}\right)-2}$,

here $\quad Y_{s}=e \phi_{s} / k_{0} T ; \quad Y_{s}^{*}=e \phi_{S}^{*} / k_{0} T \quad$ are the nondimensional potentials, $\delta_{p}=\Delta p_{0} / p_{0}$ and $\Delta p_{0}$ are the hole injection degree and the excess hole concentration, generated by the radiation, respectively. The parameter $\lambda$ is as follows: $\lambda=\sqrt{p_{0} / n_{0}}$. Let us express the equilibrium carrier concentration through the Fermi integrals [9]:

$$
\begin{aligned}
& n_{0}=4 \pi\left(2 m_{e}^{*} \frac{k_{0} T}{h^{2}}\right)^{3 / 2} F_{1 / 2}\left(\frac{E_{\mathrm{F}}}{k_{0} T}\right), \\
& p_{0}=4 \pi\left(2 m_{h}^{*} \frac{k_{0} T}{h^{2}}\right)^{3 / 2} F_{1 / 2}\left(-\frac{E_{\mathrm{F}}+E_{g}}{k_{0} T}\right),
\end{aligned}
$$

where $m_{e}^{*}=0.05 m_{0}, \quad m_{h}^{*}=0.4 m_{0}[5]$ are the effective masses of electrons and holes, respectively, $E_{\mathrm{F}}$ is the Fermi energy computed from the bottom of the conduction band.

The photon flux is decreased with the depth of a sample as $N(z)=N_{0} \exp (-K z)$, whereas the velocity of the electron-hole pair generation is $g(z)=-d N / d z=N_{0} K \exp (-K z)$ (here $N_{0}$ is the number of photons on the unit of the area per unit of time). Therefore, the excess concentration on stationary irradiation may be found by integration:

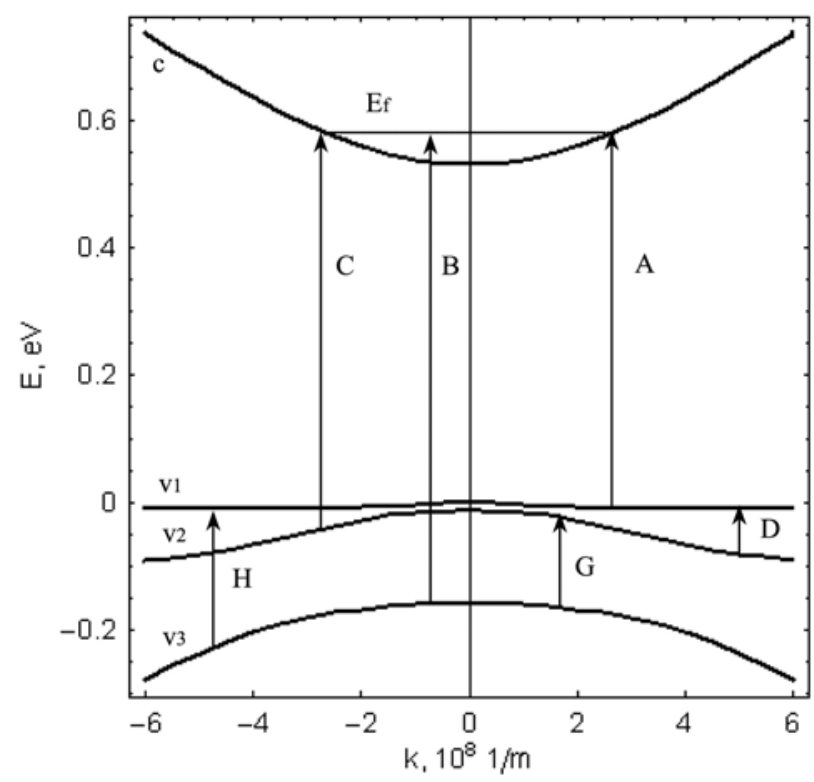

Fig. 1. Energy bands of $\mathrm{Cd}_{3} \mathrm{P}_{2}$ for $E_{\mathrm{g}}=0.53 \mathrm{eV}, \Delta=0.15 \mathrm{eV}$, $P=6.7 \cdot 10^{-10} \mathrm{eV} \mathrm{m}, \delta=0.023 \mathrm{eV}, d=0, \eta=0.99, E_{\mathrm{F}}-E_{\mathrm{g}}=$ $0.06 \mathrm{eV}$.

(C) 2006, V. Lashkaryov Institute of Semiconductor Physics, National Academy of Sciences of Ukraine 


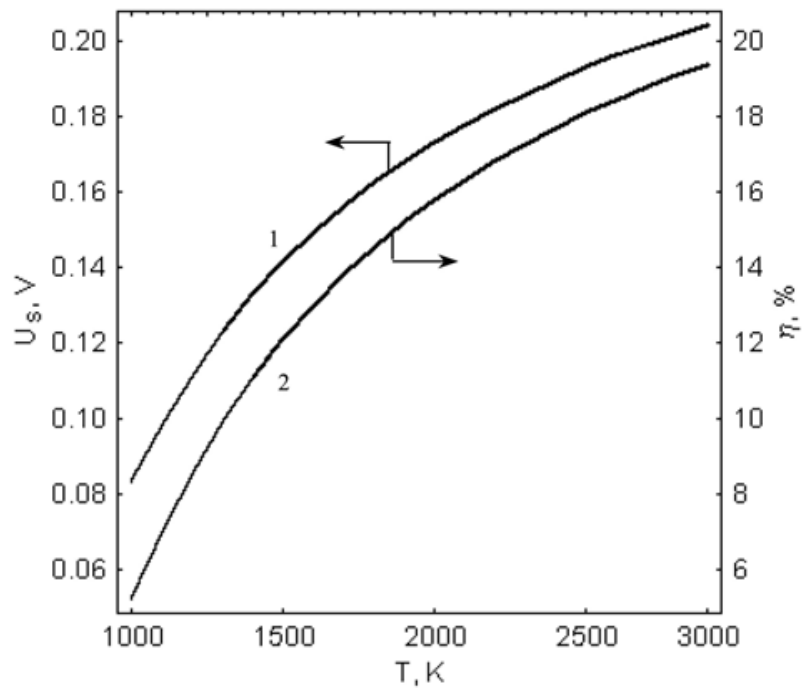

Fig. 3. Theoretical dependences of the photovoltage (1) and efficiency (2) vs the radiation temperature for $\mathrm{Me}-n-\mathrm{Cd}_{3} \mathrm{P}_{2}$ system (the temperature of material is $300 \mathrm{~K}$ ).

Now it is possible to determine the surface potential on irradiation and photovoltage of the spacecharge region, respectively, having solved Eq. (6) relative to $\phi_{s}^{*}$. Fig. 3 shows these results (curve 1).

The general thermodynamical approach was used to determine the maximum theoretical value of the converter efficiency [8]:

$\eta=\frac{E_{m} N_{h v}}{E_{c}}$.

Here $E_{m} \approx e\left(U_{s}-\frac{A k_{0} T}{e} \ln \left[\frac{e U_{s}}{A k_{0} T}+1\right]-\frac{A k_{0} T}{e}\right)$

the attainable energy as a result of the absorption of one photon, if the barrier and external electric circuit had been optimally coordinated ( $A$ is the index of ideality). Let it be $A=1$ in these calculations, because the maximal efficiency is interesting in fact. $N_{h v}=\int_{v_{1}}^{v_{2}} N d v$ is the total number of photons on the unit area per unit of time in $\left[v_{1} ; v_{2}\right]$ frequency band; $E_{c}=\int_{v_{1}}^{v_{2}} h v N d v$ is the full surface density of the radiation.

Infrared radiation causes heating the material of converter. This affects badly the converter output characteristics. Present theoretical studies have also shown that the temperature increase leads to detectable decrease of the photovoltage and efficiency values (see Fig. 4). Herewith the dependence of the bandgap value is one of the negligible factors [10].

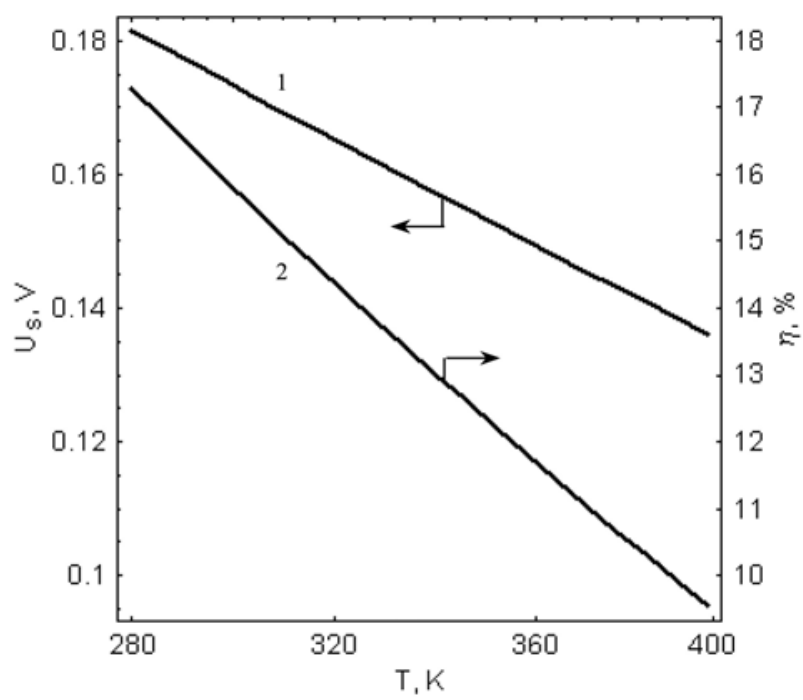

Fig. 4. Theoretical dependences of the photovoltage (1) and efficiency (2) vs the temperature of semiconductor material for $\mathrm{Me}-n-\mathrm{Cd}_{3} \mathrm{P}_{2}$ system (the radiation temperature is $2000 \mathrm{~K}$ ).

Besides, the impurities and vacancies must interact with each other much more intensively with temperature increasing. Furthermore, the temperature increase can vastly accelerate the aging process associated with the slow "annealing" of non-equilibrium vacancies. Such aging of the crystal results in lowering the Fermi level and the electron concentration more than twice for $\mathrm{Cd}_{3} \mathrm{P}_{2}$ at the same time [10]. For these reasons, the preventive measures of the converter heating will be rather necessary for this material.

\section{Conclusions}

The main results of the present investigation are as follows:

1. The set of the material parameters of $\mathrm{Cd}_{3} \mathrm{P}_{2}$ allows to more effectively use the converter for the transformation of infrared radiation into the electricity for the operating temperature range $(1000 \ldots 2500 \mathrm{~K})$ of the heat source.

2. The theoretical values of photovoltage and efficiency of the $\mathrm{Me}-n-\mathrm{Cd}_{3} \mathrm{P}_{2}$ Schottky diode are quite comparable with those for well-known diodes based on classical $p-n$ junctions.

3. The reliable thermal protection of converter (in the vicinity of the room temperature point) is rather requisite because of numerous vacancies in the crystalline structure of $\mathrm{Cd}_{3} \mathrm{P}_{2}$ and the aging phenomena.

These conclusions are quite preliminary, because, firstly, they are based just on theoretical calculations. Secondly, the complete and non-simplified theoretical description of the metal-semiconductor contacts is a very complex problem. For these reasons, the experimental confirmation is necessary, too. That is why the final decision about possibility to use cadmium phosphide for 
infrared converters may be reliable only after practical experiments. Nevertheless, the present results show these experiments are undoubtedly perspective.

\section{References}

1. K. Sieranski, J. Szatkowski, and J. Misiewicz, Semiempirical tight-binding structure of $\mathrm{II}_{3} \mathrm{~V}_{5}$ semiconductors: $\mathrm{Cd}_{3} \mathrm{P}_{2}, \mathrm{Zn}_{3} \mathrm{P}_{2}, \mathrm{Cd}_{3} \mathrm{As}_{2}$, and $\mathrm{Zn}_{3} \mathrm{As}_{2}$ // Phys. Rev. 50(11), p. $7331-7337$ (1994).

2. G. Chuiko, N. Don, O. Dvornik, V. Ivchenko, A. Sergeev, Simple inverted band structure model for cadmium arsenide $\left(\mathrm{Cd}_{3} \mathrm{As}_{2}\right)$ // Moldavian $\mathrm{J}$. Phys. Sci. 2(1), p. 88-94 (2003).

3. E.O. Kane, Band structure of indium antimonide // J. Phys. Chem. Solids 1, p. 249-261 (1957).
4. H. Kildal, Band structure of $\mathrm{CdGeAs}_{2}$ near $\mathrm{k}=0$ // Phys. Rev. 10(12), p. 5082-5087 (1974).

5. M.J. Gelten, A. van Lieshout, C. van Es, F.A.P. Blom, Optical properties of $\mathrm{Cd}_{3} \mathrm{P}_{2} / /$ Solid State Phys. 11, p. 227-237 (1978).

6. T.S. Moss, G.J. Burrell, B. Ellis, Semiconductor optoelectronics. Butterworth's, London, 1973.

7. V.F. Kisylev, S.N. Kozlov, A.V. Zoteev, Fundamental of solid state surface physics. Moscow State University, Moscow, 1999 (in Russian).

8. S.M. Sze, Semiconductor device physics. Mir, Moscow, 1984 (in Russian).

9. R. Smith, Semiconductors. Mir, Moscow, 1982 (in Russian).

10. V.B. Lazarev, V.Y. Greenberg, V.D. Rymiantsev, Semiconductor compounds of $A^{I I} B^{V}$ group. Nauka, Moscow, 1978 (in Russian). 